\title{
APPROXIMATION BY HOMEOMORPHISMS AND SOLUTION OF P. BLASS PROBLEM ON PSEUDO-ISOTOPY
}

\section{W. HOLSZTYŃSKI}

ABstract. For every map of $f: S^{1} \rightarrow S^{1}=\{z \in C:|z|=1\}$ of degree 1 , existence of a pseudo-isotopy $h: S^{1} \times I \rightarrow R=\{z \in C:|z| \geqq 1\}$ such that $h(z, 0)=z$ and $h(z, 1)=f(z)$ is established. On the other hand (i) maps of $I^{n}$ into $I^{n} \times 0 C E^{n+1}$ cannot be, in general, uniformly approximated by homeomorphic embeddings of $I^{n}$ in $E^{n+1}$ for $n>1$, and (ii) maps of $S^{n}$ into $S^{n} C E^{n}$ of degree 1 cannot be, in general, extended to a pseudo-isotopy of $S^{n}$ into $E^{n+1}$.

P. Blass asked me: Can every mapping $g: S^{n} \rightarrow S^{n}$ of degree 1 be obtained by a pseudo-isotopy in Euclidean space $E^{n+1}$ from an embedding? Does there hold an analogous assertion for mappings of $S^{n}$ into itself of other degree?

We will show that the answer is positive for $n=1$ (see $\$ 1$ ) and negative for $n>1$ (see $\$ 4$ ).

1. 1-dimensional case of a map of degree 1. First we will describe the most intuitive case. Some more general and stronger results are contained in $\$ 2$.

Let $C$ be the complex plane,

$$
\begin{gathered}
S^{1}=\{z \in C:|z|=1\}, \\
R=\{z \in C:|z| \geqq 1\} .
\end{gathered}
$$

(1.1) Theorem. Let $f: S^{1} \rightarrow S^{1}$ be a map of degree 1. Then there exists a homotopy $F: S^{1} \times I \rightarrow R$ such that $F \mid S^{1} \times\{t\}$ is a homeomorphic embedding for every $t \in I \cdot\{1\}$, and $F(z, 1)=f(z)$ for every $z \in S^{1}$.

Proof. Instead of pair $\left(R, S^{1}\right)$ we will consider the homeomorphic pair $\left(S^{1} \times E^{+}, S^{1} \times\{0\}\right)$, where $E^{+}$is the set of all nonnegative reals. Let $h: S^{1} \times I \rightarrow S^{1}$ be a homotopy such that $h(z, 0)=2$ and $h(2,1)$ $=f(z)$ for $z \in S^{1}$. Next, let

$$
u(z, t)=h(z, t) z^{-1} \text { for }(z, t) \in S^{1} \times I .
$$

Then $u\left(S^{1} \times\{0\}\right)=1$, so that there exists a mapping $v: S^{1} \times I \rightarrow E^{+}$

Received by the editors January 7, 1970.

AMS 1970 subject classifications. Primary 57A35, 57A05; Secondary 55C25.

Key words and phrases. Pseudo-isotopy, generalizated pseudo-isotopy, isotopy domination, approximation by homeomorphisms, maps of degree 1 , sphere $S^{1}$ and $S^{n}$, complex plane $C$, Euclidean space $E^{n+1}$. 
such that $u=e^{2 \pi i \cdot v}$. Homotopy $F_{0}: S^{1} \times I \rightarrow S^{1} \times E^{+}$given by formula $F_{0}=h \Delta v$ i.e. $F_{0}(z, t)=(h(z, t), v(z, t))$ has the following two properties: if $t<1$ and $z_{1} \neq z_{2}$ and $h\left(z_{1}, t\right)=h\left(z_{2}, t\right)$ then $u\left(z_{1}, t\right) \neq u\left(z_{2}, t\right)$ and $F_{0}\left(z_{1}, t\right) \neq F_{0}\left(z_{2}, t\right)$. Thus $F_{0} \mid S^{1} \times\{t\}$ is a homeomorphic embedding for $t<1$. The second property is:

$$
F_{0}(z, 1)=(f(z), v(z, 1)) .
$$

Thus the required homotopy $F: S^{1} \times I \rightarrow S^{1} \times E^{+}$can be given by

$$
\begin{aligned}
F(z, t) & =F_{0}(z, t / 2) & & \text { for } 0 \leqq t \leqq \frac{1}{2}, \\
& =(f(z),(2-2 t) \cdot v(z, 1)) & & \text { for } \frac{1}{2} \leqq t \leqq 1 .
\end{aligned}
$$

Remark. A homotopy $F$ on $X$ such that $F \mid X \times\{t\}$ is a homeomorphicembedding for $0 \leqq t<1$ is said to be a pseudo-isotopy (compare $F$ from Theorem (1.1)).

2. 1-dimensional case of a map of degree $n$. We will give a generalization of pseudo-isotopy.

(2.1) Definition. Given topological spaces $X, Y$, a mapping $g: X \rightarrow Y$ is isotopically dominated by a mapping $f: X \rightarrow Y$ iff there exists a homotopy $F: X \times I \rightarrow Y$ such that

(i) $F(x, 0)=f(x)$ and $F(x, 1)=g(x)$.

(ii) If $0 \leqq t<1$ and $f(p) \neq f(q)$ then $F(p, t) \neq F(q, t)$ for every $p$, $q \in X$.

(iii) If $\frac{1}{2} \leqq t<1$ then $F(p, t)=F(q, t)$ iff $F\left(p, \frac{1}{2}\right)=F\left(q, \frac{1}{2}\right)$, for every $p, q \in X$.

The homotopy $F$ will be called a pseudo-isotopy. In the case of a homeomorphic embedding $f$ and compact $X$ the homotopy $F$ is a pseudo-isotopy in the usual sense.

Let us remark that in such a case also the homeomorphic embedding $f$ is isotopically dominated by $g$ (we can define a respective pseudoisotopy $G$ by $G(x, t)=F(x, 1-t))$.

(2.2) Theorem. If, for $f, g: S^{1} \rightarrow S^{1}, R$ ord $f=$ ord $g$ then $g$ is isotopically dominated by $f$ in $R$.

Proof. Let $h: S^{1} \times I \rightarrow S^{1}$ be a homotopy that connects $f$ and $g$, i.e. $h(z, 0)=f(z)$ and $h(z, 1)=g(z)$. Next, let

$$
u(z, t)=h(z, t)(f(z))^{-1} \text { for } \quad(z, t) \in S^{1} \times I \quad \text { (compare (1.2)). }
$$

Then $u\left(S^{1} \times\{0\}\right)=1$ so that there exists a mapping $v: S^{1} \times I \rightarrow E^{1}$ into the real line $E^{1}$ such that $u=e^{2 \pi i \cdot v}$. Then the desired pseudoisotopy $F: S^{1} \times I \rightarrow R$ is given by 


$$
\begin{aligned}
F(z, t) & =v^{\prime}(z, 2 t) \cdot h(z, 2 t) & & \text { for } 0<t<\frac{1}{2} \\
& =\left(2(1-t) v^{\prime}(z, t)+2 t-1\right) \cdot g(z) & & \text { for } \frac{1}{2} \leqq t \leqq 1,
\end{aligned}
$$

where $v^{\prime}(z, t)=1+v(z, t)-\inf _{x \in s^{1}} v(x, t)$.

Indeed, $F$ is a well-defined mapping and condition (i) holds. Next, if $f(p) \neq f(q)$ and $h(p, t)=h(q, t)$ then $u(p, t) \neq u(q, t)$, and consequently $v^{\prime}(p, t) \neq v^{\prime}(p, t)$. But if $h\left(p, t^{\prime}\right) \neq h\left(q, t^{\prime}\right)$ or $v^{\prime}\left(p, t^{\prime}\right) \neq v^{\prime}\left(q, t^{\prime}\right)$ for $t^{\prime}=\min (2 t, 1)$ and $t<1$, then $F(p, t) \neq F(q, t)$. Thus condition (ii) holds. It is easy to see that condition (iii) also holds.

(2.4) Corollary. If $g: S^{1} \rightarrow S^{1}$ is a mapping of order 1 then there exists a homotopy $F: S^{1} \times I \rightarrow R$ such that

$\left.{ }^{*}\right) F(z, 0)=z$ and $F(z, 1)=g(z)$ for every $z \in S^{1}$,

${ }^{(* *)} F \mid S^{1} \times\{t\}$ is a homeomorphic embedding for $0 \leqq t<1$.

Looking for $F \mid S^{1} \times\left[\frac{1}{2} ; 1\right]$ at $(2.3)$ it is easy to obtain the following

(2.5) Corollary. Let ord $f=\operatorname{ord} g$ for $f, g: S 1 \rightarrow S 1$. Then there exist $f_{1}: S^{1} \rightarrow S^{1} \times I$ and $f_{2}: f_{1}\left(S^{1}\right) \rightarrow S^{1}$ such that $f=f_{2} \circ f_{1}$ and $g=p \cdot f_{1}$, where $p=S^{1} \times I \rightarrow S^{1}$ is the projection $(p(z, t)=z)$.

(2.6) Corollary. If ord $g=1$ for $g: S^{1} \rightarrow S^{1}$, then there exists a homeomorphic embedding $f_{1}=S^{1} \rightarrow S^{1} \times I$ such that $g=p \circ f_{1}$.

3. Approximations by homeomorphisms. Let $Q^{n}=\left\{x \in E^{n}:|x| \leqq 1\right\}$, $S^{n-1}=\dot{Q}^{n}$ and let $\varphi: S^{n-1} \rightarrow S^{n-1} \times\{0\} \subset E^{n+1}$ be a continuous mapping. Next let $X_{1}$ be a space obtained from $Q^{n}$ by identification of points $x, x^{\prime}$ such that $\varphi(x)=\varphi\left(x^{\prime}\right)$ and let $X_{2}$ be a space obtained from $S^{n-1} \times I$ by identification of points $(x, 0)$ and $(\varphi(x), 1)$. Then $X_{1}, X_{2}$ are the compact metrizable spaces such that

$$
H_{n-1}\left(X_{1}\right)=Z_{k} \text { and } H_{n-1}\left(X_{2}\right)=Z_{k-1}
$$

where $k=\operatorname{ord} \varphi$ (we shall consider Cech homology theory).

(3.1) THEOREM. Under the assumption $\mid$ ord $\varphi \mid>1$, there does not exist a sequence of homeomorphic embeddings of $Q^{n}$ into $E^{n+1}$ which is uniformly convergent to a mapping $g: Q^{n} \rightarrow E^{n+1}$, such that $g(x)=(\varphi(x), 0)$ for $x \in S^{n-1}$ and $g^{-1}\left(S^{n-1} \times\{0\}\right)=S^{n-1}$.

Proof. Let $f: Q^{n} \rightarrow E^{n+1}$ be a homeomorphic embedding of $Q^{n}$ into $E^{n+1}$ such that

$$
\epsilon=\epsilon(f)=\max _{x \in Q^{n-1}}|f(x)-g(x)|<1 .^{1}
$$

\footnotetext{
${ }^{1}$ In fact, we think that there does not exist $\operatorname{such} f$.
} 
Then we define $h_{f}: X_{1} \rightarrow E^{n+1}$ as follows

$$
\begin{aligned}
h_{f}(x) & =f\left(\frac{x}{1-\epsilon}\right) \quad \text { for }|x| \leqq 1-\epsilon \\
& =\frac{1-|x|}{\epsilon} \cdot f\left(\frac{x}{|x|}\right)+\left(1-\frac{1-|x|}{\epsilon}\right) \cdot g(x) .
\end{aligned}
$$

Now, if for a sequence $f_{1}, f_{2}, \cdots, \epsilon=\epsilon\left(f_{n}\right) \rightarrow 0$ then the mappings $h_{f}: X_{1} \rightarrow R^{n+1}$ are arbitrarily fine (i.e. under a metric in $X_{1}$ the mappings $h_{f_{n}}$ are $\delta_{n}$-mappings with $\left.\delta_{n} \rightarrow 0\right)$. For this reason $H_{n-1}\left(h_{f}\left(X_{1}\right)\right)$ contains a cyclic element of order $k=\operatorname{ord} \varphi$, for an embedding $f$ (to prove it see for instance [1, p. 39] and [2]). But $h_{f}\left(X_{1}\right)$ is a subspace of $E^{n+1}$. This contradiction shows the truth of the theorem.

(3.2) Theorem. Let $g: S^{n-1} \times I \rightarrow E^{n+1}$ be a mapping such that $g(x, 0)=(x, 0), g(x, 1)=(\varphi(x), 0)$ for every $x \in S^{n-1}$, and $g^{-1}\left(S^{n-1} \times\{0\}\right)$ $=S^{n-1} \times\{0,1\}$. Then, under the assumption $\mid$ ord $\varphi-1 \mid>1$, there does not exist a sequence of homeomorphic embeddings of $S^{n-1} \times I$ into $E^{n+1}$ which is uniformly convergent to $g$.

ProOF. Let $f: S^{n-1} \times I \rightarrow E^{n+1}$ be a homeomorphic embedding such that

$$
\epsilon=\epsilon(f)=\max _{(x, t) \in S^{n-1} \times I}|f(x, t)-g(x, t)|<\frac{1}{2} .
$$

Then we define $h_{f}: X_{2} \rightarrow E^{n+1}$ as follows:

$$
\begin{aligned}
h_{f}(x, t) & =f(x, t) & & \text { for } \epsilon \leqq t \leqq 1-\epsilon, \\
& =\frac{t}{\epsilon} f(x, t)+\left(1-\frac{t}{\epsilon}\right) g(x, t) & & \text { for } 0 \leqq t \leqq \epsilon, \\
& =\frac{1-t}{\epsilon} f(x, t)+\frac{t-1+\epsilon}{\epsilon} g(x, t) & & \text { for } 1-\epsilon \leqq t \leqq 1 .
\end{aligned}
$$

Now we can repeat the arguments from the proof of Theorem (3.1).

4. $n$-dimensional case, $n \geqq 2$. Let $S^{n}$ be the unit sphere of Euclidean space $E^{n+1}=E^{n} \times E^{1}$, and let $g: S^{n} \rightarrow S^{n}$ be given by

$$
\begin{aligned}
& g(x, t)=(s \cdot x, 2 t+1) \quad \text { for }-1 \leqq t \leqq 0 \text { and } s=\frac{1-(2 t+1)^{2}}{|x|}, \\
&=(s \cdot \varphi(x), 1-2 \min (t, 1-t)) \\
& \text { for } 0 \leqq t \leqq 1 \text { and } s=1-4\left(t-\frac{1}{2}\right)^{2}
\end{aligned}
$$


where $\varphi: S^{n-1} \rightarrow S^{n-1}$ is a mapping of order $\neq 0,1$. It is easy to see that ord $g=1$ i.e. that $g$ is homotopic to the identity mapping. But there does not exist a pseudo-isotopy for $g$ i.e. such a homotopy $F: S^{n} \times I$ $\rightarrow E^{n+1}$ that $F \mid S^{n} \times\{t\}$ is a homeomorphism for $0 \leqq t<1$ and that $F(x, 1)=g(x)$ for every $x \in S^{n}$. Furthermore, let

$$
P_{1}=\left\{(x, t) \in S^{n}: t \geqq \frac{1}{2}\right\}, \quad P_{2}=\left\{(x, t) \in S^{n}:|t| \leqq \frac{1}{2}\right\}
$$

and $g_{i}=g \mid P_{i}$ for $i=1,2$. We denote also by $p: E^{n+1} \rightarrow E^{n}$ the projection given by $p(x, t)=x$. Then the following lemmas hold; these are the consequences of the result of $\S 3$.

(4.1) Lemma. If $\mid$ ord $\varphi \mid>1$ then there does not exist a sequence of homeomorphic embeddings $f_{n}: P_{1} \rightarrow E^{n+1}$ which is uniformly convergent $\operatorname{tog}_{1} \mid P_{1}$.

(4.2) Lemma. If $\mid$ ord $\varphi-1 \mid>1$ then there does not exist a sequence of homeomorphic embeddings $f_{n}: P_{2} \rightarrow E^{n+1}$ which is uniformly convergent to $g_{2} \mid P_{2}$.

(4.3) CoROllaRY. For every $n \geqq 2$ there exists a mapping $g: S^{n} \rightarrow S^{n}$ $\subseteq E^{n+1}$ of order 1 , that is not isotopically dominated by a homeomorphic embedding.

\section{REFERENCES}

1. K. Borsuk, Theory of retracts, Monografie Mat., Tom 44, PWN, Warsaw, 1967. MR 35 \#7306.

2. W. Holsztynski and S. Iliadis, Approximation of multi-valued by single-valued mappings and some applications, Bull. Polon. Acad. Sci. Sér. Sci. Math. Astronom. Phys. 16 (1968), 765-769. MR 39 \#929.

University of Michigan, ANn Arbor, Michigan 48104. 